\title{
Comparative study of the in vitro and in vivo characteristics of cationic and neutral liposomes
}

This article was published in the following Dove Press journal:

International Journal of Nanomedicine

30 November 2011

Number of times this article has been viewed

\section{Wei Zhao \\ Song Zhuang \\ Xian-Rong Qi}

Department of Pharmaceutics, School of Pharmaceutical Sciences, Peking University, Beijing, People's Republic of China
Correspondence: Xian-Rong Qi

Department of Pharmaceutics, School of Pharmaceutical Sciences, Peking University, Xueyuan Road 38, Beijing I0019I, People's Republic of China

$\mathrm{Tel}+86 \quad 108280 \quad 1584$

Fax +86 I0 $8280 \quad I 584$

Email qixr200I@yahoo.com.cn
Abstract: Neutral liposomes (NLP) exhibit preferential localization in solid tumors based on the enhanced permeation and retention (EPR) effect. Cationic liposomes (CLP) have a propensity for localizing in newly formed tumor vessels and they have a potentially enhanced antitumor effect. However, an increased amount of cationic lipids in liposomes also induces aggregation through electrostatic interactions between the liposomes and the anionic species in the circulation, which results in a reduced EPR effect. Consequently, it is important to investigate the characteristics of liposomes with different surface potentials in vitro to achieve an optimal intratumoral distribution and antitumor effect in vivo. In this study, the authors evaluated the characteristics of doxorubicin (DOX)-loaded NLPs, CLPs, polyethylene glycol (PEG)-modified NLPs (NLP-PEG), and PEGylated CLPs (CLP-PEG) (ie, encapsulation efficacy, zeta potential, size, membrane fluidity, aggregation in serum, and uptake of liposomes into rat aortic endothelial cells (RAECs)) to further understand their influences on the pharmacokinetics, biodistribution, and antitumor therapy in vivo. The results showed that increased amounts of cationic lipids resulted in severe liposome aggregation in the presence of serum, yet it did not alter the membrane fluidity to a large extent. The uptake of liposomes into RAECs, visualized by confocal fluorescence microscopy, confirmed the rapid uptake of CLP by the endothelial cells compared with NLP. However, the pharmacokinetics, biodistribution and anticancer efficacies of these liposomes in vivo revealed that the CLP with highly positive surface potentials exhibited reduced circulation times and poor distribution in tumors. The NLP-PEG exhibited the highest anticancer efficacy; CLP-PEG, the second highest; and CLP with the most positive surface potential, the lowest. These phenomena were mostly due to the rapid aggregation in serum and subsequent accumulation in the lungs upon the intravenous injection of the CLP. Caution should be exercised when chemotherapeutic drugs are loaded into CLP for tumor therapy.

Keywords: cationic liposomes, PEGylation, electrostatic interaction, aggregation, anticancer efficacy, biodistribution

\section{Introduction}

Liposomes are widely recognized as potential anticancer chemotherapeutic agent carriers. Large arrays of drugs have been encapsulated in neutral or sterically stable liposomes for cancer treatment. Some anticancer liposomal drug delivery systems are already approved for clinical use. ${ }^{1}$ Neutral liposomes (NLP) exhibit preferential localization in solid tumors based on the enhanced permeation and retention (EPR) effect, which relies on the gradual passive accumulation of liposomes in the tumor. ${ }^{1,2}$ The liposomes modified with polyethylene glycol (PEG) (Stealth ${ }^{\circledR}$ liposomes; Liposome Technology, Inc., Menlo Park, CA) may enhance the EPR effect, which relies on extending the circulation time and escaping from the reticuloendothelial 
system (RES). ${ }^{3,4}$ The PEGylation of liposomes also prevents aggregation and aids the formation of small, monodisperse particles.

Cationic liposomes (CLP) are commonly used for delivering genes by utilizing the electrostatics between positively charged lipids and negatively charged nucleic acids. Recent studies have shown that CLP have a propensity for localizing in newly formed tumor vessels; however, neither the mechanism behind this selectivity nor the optimal formulation to maximize this effect has been identified. There are anionic sites in the angiogenic endothelial cells (such as anionic phospholipids, proteoglycans, hyperglycosylated and hypersialylated membrane proteins, ${ }^{5-7}$ and so forth) that may be the selective targeting sites. It has recently been reported that paclitaxel, doxorubicin, and oxaliplatin encapsulated in CLP exhibited improved antitumor efficacy associated with impaired function of the tumor microvasculature. ${ }^{8-11}$ Campbell et al ${ }^{12}$ stated that a positive charge on liposomes is required to enhance their interactions with the glycoprotein layer of the endothelium. However, unshielded CLP or lipoplexes themselves are well known to possess poor physicochemical characteristics for systemic delivery. Their positive surface charge leads to nonspecific interactions with the anionic species in the blood, which results in rapid clearance from the circulation by the $\mathrm{RES}^{13-15}$ and a reduced EPR effect. ${ }^{10,16,17}$ It was hypothesized that an increased amount of cationic lipids in liposomes may lead to a higher affinity for the angiogenic endothelial cells, which may enhance the anticancer effects. However, it may also enhance the aggregation of CLP in the bloodstream through electrostatic interactions with the anionic species in the blood and enhancing uptake by the RES. The two effects are interrelated in adverse and/or more complicated form to affect the fate of CLP in vivo. Consequently, it is important to investigate the in vitro and in vivo characteristics of liposomes with different surface potentials to achieve an optimal intratumoral distribution and antitumor effect in vivo.

In this study, the authors evaluated doxorubicin (DOX)loaded NLP, CLP, PEGylated NLP (NLP-PEG), and PEGylated CLP (CLP-PEG) by investigating their characteristics in vitro (ie, encapsulation efficacy, zeta potential, size, membrane fluidity, and aggregation in serum) to further understand their influences on tumor therapy. Using confocal fluorescence microscopy, the authors confirmed the rapid uptake of CLP-PEG and CLP by endothelial cells in vitro, compared with NLP. The pharmacokinetics, distribution, and anticancer efficacies of the NLP and CLP in tumor-bearing mice were also investigated. The in vivo results revealed that the PEGylated liposomes had better anticancer efficacy.

\section{Materials and methods Materials}

L- $\alpha$-Dipalmitoylphosphatidylcholine (DPPC) was purchased from NOF Corporation (Tokyo, Japan) and cholesterol (Chol) was obtained from Wako Pure Chemical Industries, Ltd (Osaka, Japan). 33-[N-[2-(N, N-dimethylamino) ethyl]carbamoyl] cholesterol (DC-Chol) was synthesized as described. ${ }^{18}$ 1,2-Distearoyl-sn-glycero-3-phosphoethanolamine-N-[poly (ethylene glycol) ${ }_{2000}$ ] (DSPE-PEG) was purchased from Northern Lipids Inc (Burnaby, British Columbia, Canada). Zhejiang Hisun Pharmaceutical Co, Ltd (Taizhou Zhejiang, China) kindly donated the DOX. 1,6-Diphenyl-1,3,5-hexatriene (DPH) and daunorubicin hydrochloride (DAU) were purchased from Sigma-Aldrich (St Louis, MO). Newborn calf serum (NCS) was obtained from HyColone Laboratories, Inc (Logan, UT). All other chemicals were commercial products of analytical grade. The hepatocarcinoma (H22) cells and sarcoma 180 (S180) cells were a kind gift from the Pharmacology Department, School of Pharmaceutical Sciences, Peking University.

\section{Liposome preparation and characterization}

Several liposomes with a total lipid content of $20 \mu \mathrm{mol} / \mathrm{mL}$ were prepared using the film dispersion method. The ratio in each liposome denotation shown in Table 1 refers to the molar ratio of neutral lipid to cationic lipid: LP (neutral liposomes only containing DPPC without chol and DC-chol) 10:0, NLP 10:0, NLP 10:0-PEG, CLP 9:1, CLP 3:1, CLP 3:1-PEG, CLP 5:5, and CLP 5:5-PEG.

The lipids were dissolved in one aliquot of chloroform and one aliquot of methanol. The solvent was evaporated under vacuum in a rotary evaporator at $45^{\circ} \mathrm{C}$ until a thin lipid film was formed. Drying the film under vacuum for 1 hour further eliminated solvent traces. Liposomes were formed upon the addition of $150 \mathrm{mM}$ ammonium sulfate to the flask followed by bath sonication at $50^{\circ} \mathrm{C}$ for 10 minutes. The liposome dispersion was further probe sonicated in ice water for 3 minutes and extruded through a $200 \mathrm{~nm}$ polycarbonate membrane (Nuclepore Corp, Pleasanton, CA) at about $60^{\circ} \mathrm{C}$ to obtain a homogeneous liposome suspension.

DOX was loaded in the liposomes by remote loading using an ammonium sulfate gradient. Briefly, liposomes dispersed in a $150 \mathrm{mM}$ ammonium sulfate solution were loaded onto a Sephadex G-50 column (Pharmacia, Uppsala, 
Table I Liposome formulations and characteristics

\begin{tabular}{|c|c|c|c|c|c|c|c|c|}
\hline \multirow[t]{2}{*}{$\begin{array}{l}\text { Liposome } \\
\text { denotations }\end{array}$} & \multicolumn{4}{|c|}{$\begin{array}{l}\text { Liposome formulation } \\
\text { (molar ratio)* }\end{array}$} & \multirow[t]{2}{*}{$\begin{array}{l}\text { Encapsulation } \\
\text { efficiency (\%) }\end{array}$} & \multirow[t]{2}{*}{$\begin{array}{l}\text { Zeta potential } \\
(\mathrm{mV})\end{array}$} & \multirow[t]{2}{*}{$\begin{array}{l}\text { Size } \\
(\mathrm{nm})\end{array}$} & \multirow[t]{2}{*}{$\begin{array}{l}\text { Polydispersity } \\
\text { index }\end{array}$} \\
\hline & DPPC & Chol & DC-Chol & DSPE-PEG & & & & \\
\hline LP I0:0 & 100 & 0 & 0 & 0 & $N R$ & $+0.76 \pm 0.55$ & 124 & 0.398 \\
\hline NLP I0:0 & 50 & 50 & 0 & 0 & $102.7 \pm 5.3$ & $-7.31 \pm 1.74$ & $14 \mid \pm 35$ & $0.384 \pm 0.121$ \\
\hline NLP I0:0-PEG & 45 & 50 & 0 & 5 & $91.4 \pm 1.6$ & $-3.99 \pm 0.42$ & $86 \pm 4$ & $0.171 \pm 0.015$ \\
\hline CLP 9:I & 50 & 40 & 10 & 0 & $91.3 \pm 2.1$ & $+5.58 \pm 1.46$ & $131 \pm 19$ & $0.404 \pm 0.106$ \\
\hline CLP 3:I & 50 & 25 & 25 & 0 & $93.3 \pm 2.7$ & $+11.80 \pm 1.38$ & $101 \pm 11$ & $0.222 \pm 0.050$ \\
\hline CLP 3:I-PEG & 47.5 & 25 & 25 & 2.5 & $97.8 \pm 3.6$ & $+1.33 \pm 1.27$ & $99 \pm 13$ & $0.202 \pm 0.058$ \\
\hline CLP 5:5 & 50 & 0 & 50 & 0 & $92.4 \pm 7.8$ & $+24.25 \pm 1.93$ & $95 \pm 14$ & $0.299 \pm 0.078$ \\
\hline CLP 5:5-PEG & 45 & 0 & 50 & 5 & $96.0 \pm 1.2$ & $+3.82 \pm 0.98$ & $102 \pm 37$ & $0.198 \pm 0.033$ \\
\hline
\end{tabular}

Notes: *All liposomes were prepared by the same method with the same total lipid molar concentration. When loaded with DOX, the DOX concentration was $0.5 \mathrm{mg} / \mathrm{mL}$ with a DOX-to-lipid mass ratio of I:20. The particle size and zeta potential were measured in phosphate-buffered saline ( $\mathrm{pH} 7.4$ ). Data represent the mean plus or minus standard deviation $(n=3-5)$.

Abbreviations: Chol, cholesterol; CLP, cationic liposome; DC-Chol, $3 \beta-[\mathrm{N}-[2-(\mathrm{N}, \mathrm{N}-$ dimethylamino)ethyl]carbamoyl] cholesterol; DOX, doxorubicin; DPPC, L- $\alpha-$ dipalmitoylphosphatidylcholine; DSPE-PEG, I,2-Distearoyl-sn-glycero-3-phosphoethanolamine-N-[poly (ethylene glycol) ${ }_{2000}$; NLP, neutral liposome; LP, neutral liposomes without cholesterol; NR, not reported; PEG, polyethylene glycol.

Sweden) and eluted with phosphate-buffered saline (PBS; $138 \mathrm{mM}$ sodium chloride $(\mathrm{NaCl}), 2.68 \mathrm{mM}$ potassium chloride, $9.69 \mathrm{mM}$ disodium hydrogen phosphate monohydrate, $1.46 \mathrm{mM}$ potassium dihydrogen phosphate $(\mathrm{pH}$ 7.4)) to achieve an ammonium sulfate gradient. DOX was then added to the liposome suspension to attain a drug-to-lipid molar ratio of 1:20 (w/w), and the samples were incubated in a water bath at $60^{\circ} \mathrm{C}$ for 10 minutes with continual shaking to provide optimal loading conditions. The liposomes loaded with DOX were extruded through a $200 \mathrm{~nm}$ polycarbonate membrane and stored at $4^{\circ} \mathrm{C}$.

To determine the DOX encapsulation efficiency, free DOX was separated from the liposomal DOX by a Sephadex G-50 column eluted with PBS. The concentration of liposome-entrapped DOX was measured using a fluorescence spectrophotometer ( $\lambda$ ex: $480 \mathrm{~nm}$; $\lambda$ em: $590 \mathrm{~nm}$ ) (RF-5301PC; Shimadzu, Kyoto, Japan) following the destruction of the liposomes by adding acidified isopropanol ( $0.75 \mathrm{~N}$ hydrochloric acid). The encapsulation efficiency is expressed as the percentage of DOX loaded into liposomes after the removal of the free DOX by gel filtration. The particle size and zeta potential of these liposomes were analyzed by photon correlation spectroscopy and laser Doppler microelectrophoresis using a Malvern Zetasizer 3000 (Malvern Instruments Ltd, Worcestershire, UK).

\section{Liposome turbidity in NCS and saline}

The turbidity of liposome suspensions in 50\% NCS and in saline $(0.9 \% \mathrm{NaCl}$ solution) was studied by determining the absorbance at $550 \mathrm{~nm}$ of five blank liposome suspensions of NLP 10:0, CLP 9:1, CLP 3:3, CLP 5:5, or CLP 5:5-PEG containing the same lipid concentration of $16 \mu \mathrm{mol} / \mathrm{mL}$ at various time points and at room temperature $\left(27.5^{\circ} \mathrm{C}\right)$. Fifty percent
NCS was treated as a control following the previous procedure. The relative absorbance values ( $\%$ ) were calculated according to $A_{t} / A_{0} \times 100$, where $A_{0}$ denotes the initial absorbance of the sample and $\mathrm{A}_{t}$ denotes the absorbance at a given time ( $\mathrm{t}$ ) upon mixing the liposomes with saline or with NCS.

\section{Measurement of the fluorescence polarization of liposomal membranes}

$\mathrm{DPH}$, a fluorescent probe, was dissolved in tetrahydrofuran at a $2 \mathrm{mM}$ concentration and was added to lipids dissolved in chloroform and methanol at a DPH-to-lipid molar ratio of 1:200 before a thin film formed. The liposomes were prepared by the method described at a total lipid concentration of $4 \mathrm{mM}$ in PBS. The fluorescence intensities were measured at $30^{\circ} \mathrm{C}-65^{\circ} \mathrm{C}$ with a fluorescence spectrophotometer $(\mathrm{F}-45000$; Hitachi High-Technologies Corporation, Tokyo, Japan) at $350 \mathrm{~nm}$ for excitation and $450 \mathrm{~nm}$ for emission. All measurements were performed in quartz cuvettes thermostatized by a circulating water bath (PolyScience, Niles, IL) that was connected to the cuvette holder. The fluorescence polarization $(p)$ can be calculated using the following equation:

$$
p=\frac{I_{V V}-G \times I_{V H}}{I_{V V}+G \times I_{V H}} .
$$

In this equation, $I_{V V}$ denotes the fluorescence intensities of the emitted light polarized parallel to the vertically polarized exciting light and $I_{V H}$ denotes the fluorescence intensities of the emitted light polarized perpendicular to the vertically polarized exciting light. $G=I_{H V} / I_{H H}$ is the instrumental grating factor, ${ }^{19}$ where $I_{H V}$ and $I_{H H}$ are the fluorescence intensities of the emitted light polarized perpendicular and parallel to the horizontally polarized exciting light. 


\section{Cell culture and intracellular uptake of liposomes}

The Sprague Dawley rat aortic endothelial cells (RAECs) were isolated according to the reported procedure ${ }^{20,21}$ and were cultured in RPMI 1640 medium (Gibco Invitrogen, Grand Island, NY) supplemented with $10 \%$ (v/v) heat-inactivated NCS, $200 \mathrm{U} / \mathrm{mL}$ penicillin, $100 \mathrm{U} / \mathrm{mL}$ streptomycin, and $0.11 \mathrm{mg} / \mathrm{mL}$ sodium pyruvate. Cells were maintained in a $5 \%$ carbon dioxide atmosphere at $37^{\circ} \mathrm{C}$. RAECs from passages three and four were used for all experiments.

Sterile coverslips were placed in six-well plates. RAECs were seeded at a concentration of $2 \times 10^{4}$ per $\mathrm{mL}$ in the same six-well plates. Following an incubation period of 24 hours at $37^{\circ} \mathrm{C}$ in RPMI 1640 medium containing $10 \%$ NCS, the medium was replaced with serum-free RPMI 1640 medium, which was followed by the addition of DOX-loaded liposomes or free DOX saline solution (DOX final concentration, $10 \mu \mathrm{g} / \mathrm{mL}$; lipid final concentration, $2.7 \mu \mathrm{mol} / \mathrm{mL}$ ) to each well. The cells were incubated for additional times (15 minutes, 30 minutes, 1 hour, 4 hours) with liposomes and washed with PBS three times to remove unassociated liposomes. The cells were then fixed at room temperature in 4\% paraformaldehyde for 20 minutes, which was followed by adding $0.2 \%$ Triton X-100 (Amresco LLC, OH) to disrupt the cell membranes. After washing with PBS three times, the cells were treated with diaminophenylindole (DAPI) (SigmaAldrich, St Louis, MO) to stain the cell nuclei. The coverslip from each well was mounted onto a glass microslide with $80 \%$ fluorescence-free glycerol mounting medium. Finally, the interaction of liposomes with RAECs and the intracellular uptake was observed using a confocal microscope (Leica Microsystems GmbH, Wetzlar, Germany) to visualize DAPI $(345 / 661 \mathrm{~nm})$ and DOX $(488 / 590 \mathrm{~nm})$.

\section{Animal and tumor models}

Kunming (KM) mice ( $20 \mathrm{~g}$ bw), purchased from Peking University Animal Laboratory, were chosen to establish the tumor-bearing model. In vivo experiments were carried out according to institutional and governmental guidelines. The animals were housed in cages and had free access to tap water and standard laboratory food throughout the experiments.

The H22 cells were cultured in the ascites of the KM male mice for 7-10 days. Subsequently, the ascitic fluid was removed and diluted with sterile saline to obtain an $\mathrm{H} 22$ concentration of $1 \times 10^{7} \mathrm{cell} / \mathrm{mL}$. The solid tumor model was prepared by the subcutaneous inoculation of a $0.2 \mathrm{~mL}$ suspension containing $2 \times 10^{6} \mathrm{H} 22$ cells on the right hind limb of each mouse. The H22 solid tumor-bearing mice were used in experiments for the pharmacokinetics and biodistribution studies when the tumor reached a diameter of approximately $8 \mathrm{~mm}$.

The $\mathrm{S} 180$ cell suspension was the ascitic fluid of KM mice diluted with Dulbecco's modified Eagle's medium (Gibco, Invitrogen, Carlsbad, CA). The S180 cells $\left(5 \times 10^{6} / 0.2 \mathrm{~mL}\right)$ were implanted by subcutaneous injection in the armpits of female KM mice to establish the S180 tumor model. The S180 tumor-bearing mice were used in the experiments for antitumor effects.

\section{Pharmacokinetics and biodistribution of liposomes in vivo}

The pharmacokinetics and biodistribution studies were carried out on the female KM mice bearing H22 tumors. Free DOX or DOX-loaded liposomes (NLP 10:0, CLP 9:1, CLP 5:5, or CLP 5:5-PEG) with the same DOX concentration of $0.5 \mathrm{mg} / \mathrm{mL}$ were administered to groups of six mice by intravenous injection into the tail vein at a dose of $5 \mathrm{mg} / \mathrm{kg}$ of body weight. After the injection, blood samples were collected under ether anesthesia at $0.083,0.5,1,4,8,12$, and 24 hours. The blood samples were placed in heparinized vials and centrifuged at $5000 \mathrm{rpm}$ for 10 minutes. The plasma was collected and stored at $-20^{\circ} \mathrm{C}$ until analyzed. Animals were then sacrificed by cervical dislocation immediately after blood was extracted at different time points, and the heart, liver, spleen, kidney, lung, and tumor were collected, rinsed with saline, and stored at $-20^{\circ} \mathrm{C}$ until analyzed.

An extraction protocol modified from the reported method $^{22,23}$ was used for the plasma and tissue samples. Briefly, approximately $0.2 \mathrm{~g}$ tissue was first homogenized in $400 \mu \mathrm{L}$ PBS. An aliquot of this $400 \mu \mathrm{L}$ homogenate or $200 \mu \mathrm{L}$ plasma sample was transferred to a glass centrifugal tube, which was followed by the addition of $50 \mu \mathrm{L}$ DAU $(40 \mu \mathrm{g} / \mathrm{mL})$ as an internal standard. Then, $5 \mathrm{~mL}$ organic solvent containing methanol and chloroform $(1: 4, \mathrm{v} / \mathrm{v})$ was added to the homogenate or to the plasma sample, which was subsequently vortexed for 1 minute and centrifuged at $4000 \mathrm{rpm}$ for 20 minutes. The lower organic solvent layer was collected and dried in a water bath at $40^{\circ} \mathrm{C}$ under nitrogen. Finally, it was redissolved in the mobile phase solution and stored at $-20^{\circ} \mathrm{C}$ until analyzed by high-performance liquid chromatography (HPLC).

For HPLC analysis, the $\mathrm{C}_{18}$ reverse-phase column was equilibrated with a mobile phase of methanol and water $(1: 1, \mathrm{v} / \mathrm{v})$ acidified by $85 \%$ phosphoric acid to reach a final $\mathrm{pH}$ of 3.1. The flow rate was $1 \mathrm{~mL} / \mathrm{min}$, the ultraviolet detection wavelength was $233 \mathrm{~nm}$, and the room temperature was $27^{\circ} \mathrm{C}$. 
The noncompartmental analysis was utilized for simulating the pharmacokinetics and distribution of free DOX or DOXloaded liposomes in H22 tumor-bearing mice. The mean values of each group were used in the noncompartmental analysis.

\section{Antitumor effects in vivo}

Sixty S180 tumor-bearing KM mice were randomly divided into six groups. The DOX-loaded liposomes (NLP 10:0, NLP 10:0-PEG, CLP 3:1, or CLP 3:1-PEG) with the same DOX concentration of $0.5 \mathrm{mg} / \mathrm{mL}$ were intravenously injected into the tail vein at a dose of $5 \mathrm{mg} / \mathrm{kg}$ of body weight on days 2, 4, and 6 after the tumor implantation. The DOX solution in normal saline (free DOX; the positive control) and the normal saline (the negative control) were also administered on the same schedule. The deaths of the S180 tumor-bearing mice were recorded.

\section{Statistical analysis}

Data were expressed as the mean plus or minus the standard deviation. The statistical analysis was carried out using SPSS software (v 15.0; SPSS Inc, Chicago, IL). The Kaplan-Meier survival methodology was used for analysis of the survival rate data. Other data were analyzed using the unpaired Student's $t$-test. Generally, $P<0.05$ was considered to be statistically significant.

\section{Results and discussion Liposome characterization}

Vesicle size, surface charge, liposome composition, and lipid dose are major determinants for the fate of intravenously administered liposomes. Small, PEGylated liposomes typically exhibit a decreased rate of RES uptake and increased circulation time. ${ }^{24}$

The DOX encapsulation efficiency, size, and zeta potential of the DOX-loaded liposomes are shown in Table 1. The DOX encapsulation efficiency of all liposomes was above $90 \%$, and the small differences may be due to preparation variation. The liposome sizes were approximately $85-140 \mathrm{~nm}$. The electronegativity or electropositivity at the liposome surfaces, as reflected in the zeta potential values, was affected by the liposome composition. The lower negative or positive zeta potential values were obtained for LP 10:0, NLP 10:0, and NLP 10:0-PEG. The liposomes containing the cationic lipid DC-Chol exhibited positive zeta potentials. The increase in the amount of DC-Chol undoubtedly enhanced the positive surface charge of the liposome membrane. The addition of DSPE-PEG in the liposomes reduced the absolute value of the zeta potentials. This phenomenon may be attributed to the PEG coating, which is a hydrophilic layer formed around the liposome membranes that shifts the shear plane of the liposomes, thus resulting in a decrease in the absolute value of the zeta potential. Long-chain PEG was reported to prolong the blood circulation time in vivo, which led to an increased accumulation in the tumor.

The stability of some liposomes (NLP 10:0, CLP 9:1, CLP 5:5, and CLP 5:5-PEG) stored at $4^{\circ} \mathrm{C}$ was determined every week using the encapsulation efficiencies, sizes, and zeta potentials as indicators. The encapsulation efficiencies, sizes, and zeta potentials of the liposomes fluctuated slightly over 28 days but did not significantly change, and no visible sedimentation was observed. The results suggested that the DOX-loaded liposomes would be stable for at least 28 days at $4^{\circ} \mathrm{C}$.

\section{Liposome aggregation in NCS}

There are anionic species in serum that may interact with the positively charged membranes of CLP. Thus, the interactions between anionic substances and liposome membranes may result in the aggregation of liposomes in serum. The sedimentation of agglomerates occurs readily compared with that of nonaggregated liposomes. Thus, the relative absorbance (\%) of a liposome suspension at $550 \mathrm{~nm}$ reflects the turbidity change. ${ }^{25}$ Figure 1 shows the relative absorbance (\%) of liposomes in saline and in 50\% NCS at the indicated times. The relative absorbance of liposomes in saline (Figure 1A) was measured to determine the influence of liposome self-sedimentation on aggregation. The results showed that the relative absorbance values of NLP 10:0 and CLP 9:1 changed over time, while those of CLP 3:1, CLP 5:5, and CLP 5:5-PEG were constant for up to 12 hours at room temperature. The reduction of absorbance values for NLP 10:0 and CLP 9:1 indicated that NLP 10:0 and CLP 9:1 self-aggregated due to the relatively neutral charges on the membrane surfaces. In contrast, CLP 3:1 and CLP 5:5, which have highly positive surface charges and strong self-repulsion forces, tended to be stable in saline. The CLP 5:5-PEG exhibited a similar profile to CLP 5:5 because of its outer hydrophilic PEG brush and in spite of the relatively neutral surface charges. However, in NCS (Figure 1B), CLP 3:1 and CLP 5:5 (which contained $25 \mathrm{~mol} \%$ and $50 \mathrm{~mol} \%$ of cationic lipids, respectively, and thus exhibited highly positive zeta potentials) became severely aggregated with the anionic species in serum, which was followed by a drastic fall in absorbance at 4 hours due to aggregate sedimentation. Because of their relatively negative or slightly positive charges on the membrane surfaces, the 
A

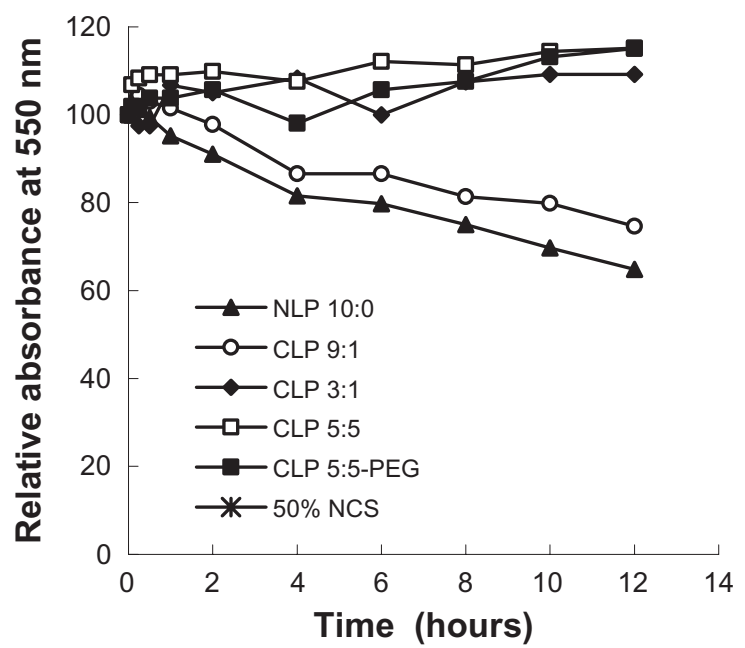

B

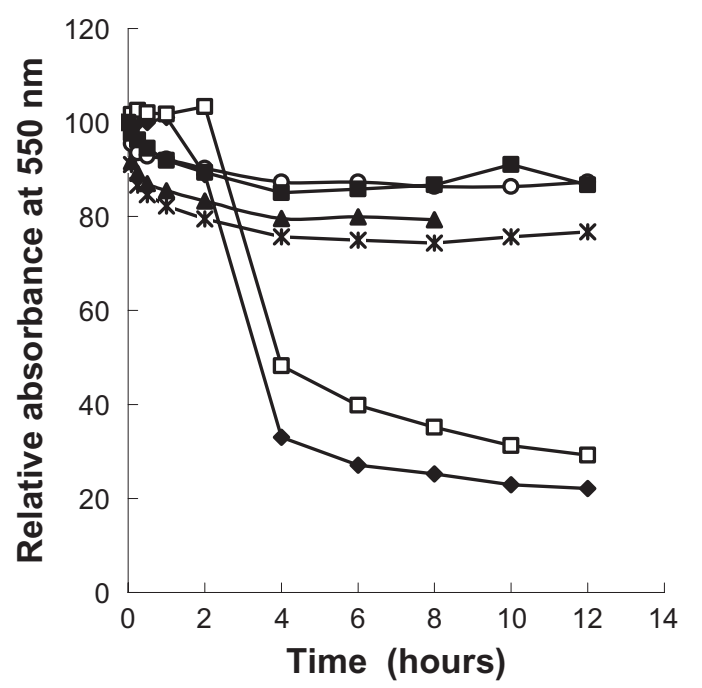

Figure I Relative absorbance values of five blank liposomes in (A) saline and (B) $50 \%$ newborn calf serum (NCS) at $550 \mathrm{~nm}$. Blank liposomes were incubated with the same volumes of NCS and saline at room temperature, and their absorbance values at $550 \mathrm{~nm}$ were measured at the indicated time points.

Note: The relative absorbance values of the five blank liposomes were calculated as $A / A_{0} \times 100$, where $A_{0}$ denotes the initial absorbance of the sample and $A_{t}$ denotes the absorbance at a given time $(\mathrm{t})$ upon mixing the liposomes with saline or with NCS.

Abbreviations: CLP, cationic liposome; NLP, neutral liposome; PEG, polyethylene glycol.

relative absorbance values of NLP 10:0, CLP 9:1, and CLP 5:5-PEG did not change significantly over time.

These results support the authors' hypothesis that there are interactions between the anionic species in serum and the CLP. Based on this sedimentation result and the stability of the liposomes at $4{ }^{\circ} \mathrm{C}$, it may be concluded that the CLP have good physical stability in saline but they interact strongly with the anionic substances in serum. Aggregations increase with the amount of cationic lipids in the membrane surface, which implies that CLP are unstable in vivo when they have a highly positive charge on the membrane surface. Modifying CLP with PEG may shade the positive charge and improve the stability of liposomes in serum and in vivo.

\section{Effect of lipid composition on the fluidity of liposomal membranes}

The results of the fluorescence polarization of DPH provide information on the orientation of the acyl chain in the liposomal bilayers. According to the Perrin-Webber equation, fluorescence polarization is correlated to the membrane fluidity near the fluorescent probe. When DPH movement is restricted in the gel state of the lipid, the liposomes exhibit high fluorescence polarization. A decrease in fluidity leads to an increase in fluorescence polarization.

The variation in the fluorescence polarization of DPH with temperature is shown in Figure 2. The fluorescence polarization of LP 10:0 (consisting of only DPPC) significantly decreased from 0.5666 at $40^{\circ} \mathrm{C}$ to 0.4703 at $45^{\circ} \mathrm{C}$, which is in accordance with the phase transition temperature of DPPC from the gel to liquid crystalline state at $41^{\circ} \mathrm{C}$. The same result had been observed by Qi et al. ${ }^{25}$ The addition of Chol and DC-Chol mitigated the fluidity changes in the liposomal membranes. The fluorescence polarization of CLP 9:1 gradually decreased with temperature from $35^{\circ} \mathrm{C}$ to $65^{\circ} \mathrm{C}$, while that of CLP 3:1 decreased and reached a plateau in the range of $45^{\circ} \mathrm{C}-55^{\circ} \mathrm{C}$, which indicates there are two different phase transition temperatures because of its varying lipid composition. Notably, in the range of $35^{\circ} \mathrm{C}-40^{\circ} \mathrm{C}$, the samples ranked in fluorescence polarization as follows: CLP 3:1-PEG > CLP 5:5-PEG > CLP 9:1 > CLP 3:1 > CLP 5:5. This indicated that, at the gel state, the increasing content of DC-Chol in the liposomal membranes led to the increase in fluidity, while the inclusion of PEG may reduce membrane fluidity. The results suggested that the addition of DC-Chol and a subsequent increase in membrane fluidity would likely lead to an increase in DOX leakage from these liposomes, while adding PEG to the membrane may reduce the leakage to some extent. The conclusion was consistent with the observation on DOX leakage in vitro in a previously published article by the authors. ${ }^{26}$

The presence of Chol exerts a profound influence on the properties of the lipid bilayers of the liposomes. The DC-Chol may have the similar influence with Chol on lipid bilayers of the liposomes. The addition of Chol is necessary in order to prevent the leakage of the encapsulated drug 


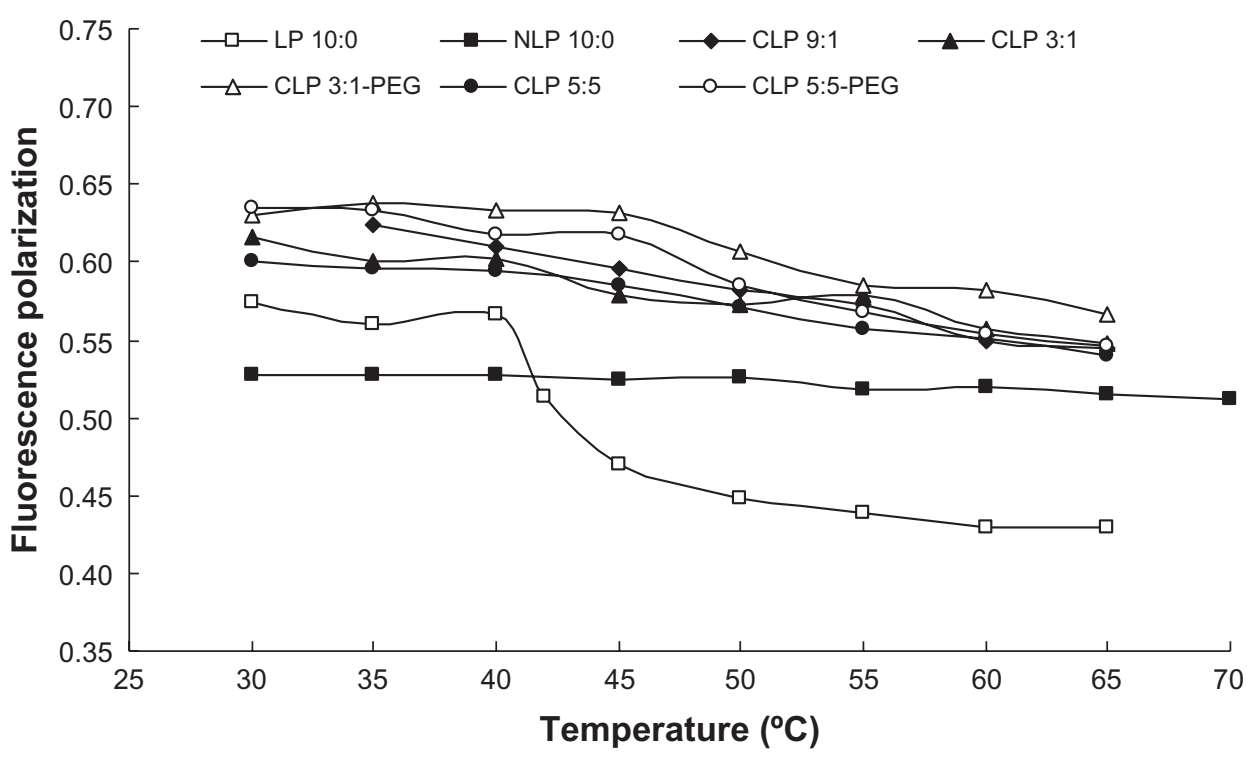

Figure 2 Relationship between fluorescence polarization of I,6-diphenyl-I,3,5-hexatriene and temperature for the liposomes with different lipid compositions. Abbreviations: CLP, cationic liposome; NLP, neutral liposome; LP, neutral liposomes without cholesterol; PEG, polyethylene glycol.

from the liposomes made from unsaturated lipids. Chol molecules fill in the free space formed because of the kink in the chain of the unsaturated lipids, and this will decrease the flexibility of the surrounding lipid chains. This interaction also increases the mechanical rigidity of fluid bilayers and decreases their lateral diffusion. In contrast, the addition of Chol to gel-phase bilayers disrupts local packing orders, increases the diffusion coefficient, and decreases the elastic modulus. One of the main reasons for the addition of Chol to liposomes made from saturated lipids is to decrease the phase transition temperature. The existence of the phaseseparated domains makes the liposomes unstable upon the phase transition temperature, and therefore the liposomes release their content upon a temperature trigger. The addition of Chol to gel-phase liposomes will increase the fluidity of the membrane and therefore all the gaps and imperfections formed in the lipid bilayers because of the heat trigger will be healed immediately; this will prevent the liposomes from leaking more of their content upon trigger, and this is undesirable.

\section{Intracellular uptake of liposomes observed by confocal fluorescence microscopy}

Confocal fluorescence microscopy was used to evaluate the liposomal localization in RAECs (see Figure 3). The red areas represent the DOX localization, the blue areas indicate the cell nuclei stained by DAPI, and the pink areas represent the localization of DOX in the nuclei.
Free DOX was immediately taken up by RAECs and localized in the nuclei and cytoplasm because of its diffusion across the plasma membrane. NLP 10:0 was taken up slowly, and there was an insignificant amount of DOX in the nuclei after 4 hours of incubation. The weak cellular uptake of NLP 10:0 by RAECs may be due to the anionic surface charges of NLP 10:0, which induce electrostatic repulsions with the cellular membrane. The CLP demonstrated an enhanced cellular uptake by RAECs compared with NLP 10:0, and their intracellular uptake varied directly with the amount of cationic lipids. This may be because of the positive charge of the CLP, which can induce electrostatic interactions with cells and facilitate DOX release into the cytosol by endosomal escape. . $^{10,27,28}$

Different lipids and cell types may result in different rates of intracellular uptake. This study confirmed that RAECs may readily take up CLP and PEGylated liposomes. There are anionic sites within angiogenic endothelial cells such as anionic phospholipids, proteoglycans, and hyperglycosylated and hypersialylated membrane proteins..$^{5-7}$ CLP were taken up via endocytosis and membrane fusion ${ }^{29}$ in a nonspecific pattern involving electrostatic interactions between the positively charged liposomes and the negatively charged cell surfaces in the absence of serum. ${ }^{12,30}$ Meanwhile, cationic lipids were reported ${ }^{31}$ to destabilize the endosomal membrane, leading to faster leakage of the loaded drug into the cytoplasm. These may be the reasons for the rapid localization of DOX from the CLP to the nuclei and cytoplasm of the endothelial cells. 


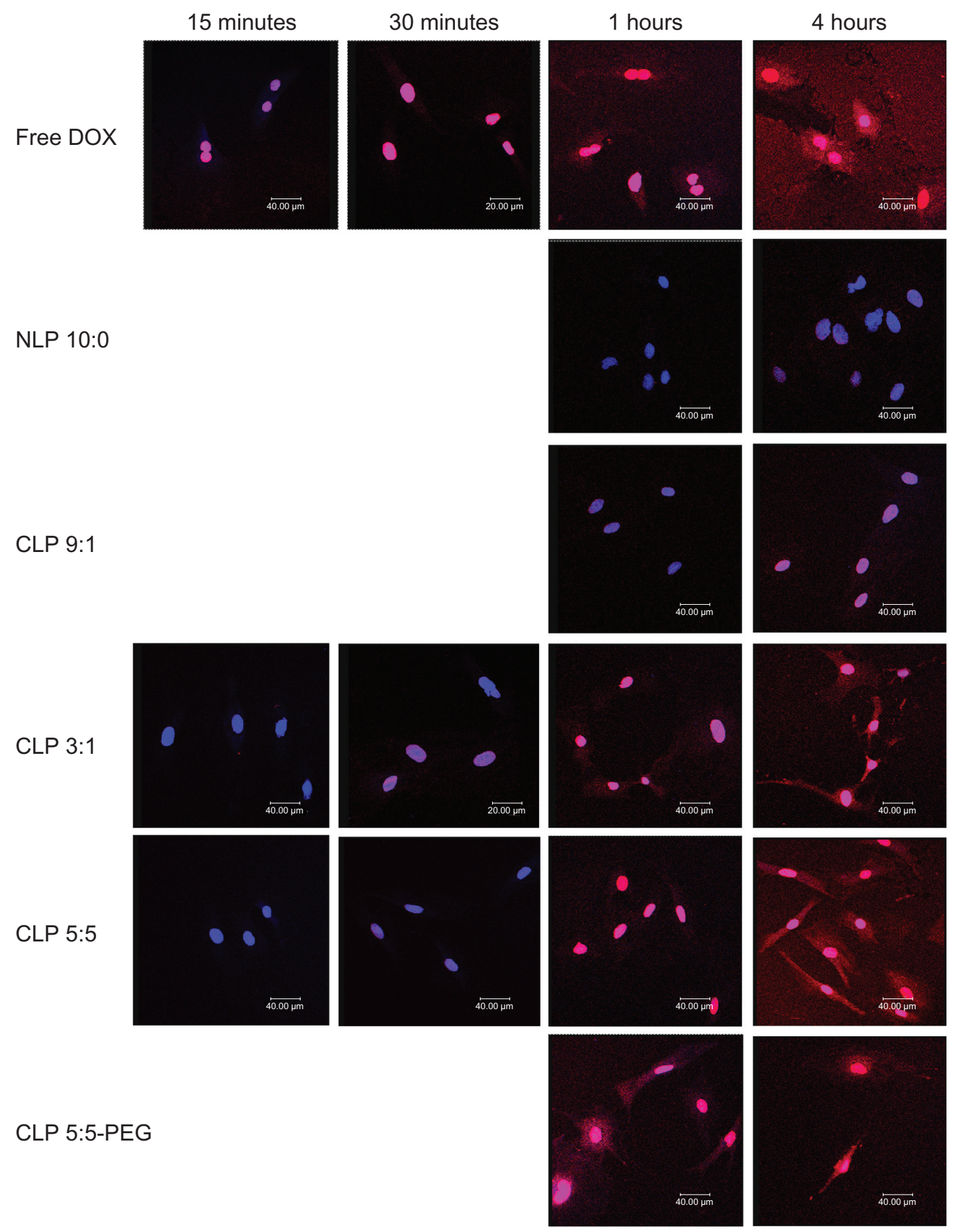

Figure 3 Confocal micrographs showing the intracellular uptake of liposomes by rat aortic endothelial cells (RAECs). RAECs were incubated with different doxorubicin (DOX)-loaded liposomes, or free DOX as a control, for 15 and 30 minutes and I and 4 hours. The nuclei were stained by diaminophenylindole (shown in blue), and DOX (shown in red) is intrinsically fluorescent.

Abbreviations: CLP, cationic liposome; NLP, neutral liposome; PEG, polyethylene glycol.

\section{Pharmacokinetics and biodistribution}

\section{studies of liposomes in tumor-bearing mice} The 24-hour profiles of the DOX concentrations in the plasma of H22 tumor-bearing mice after the intravenous injection of free DOX, NLP 10:0, CLP 9:1, CLP 5:5, or CLP 5:5PEG are shown in Figure 4. The concentration of free DOX drastically decreased within 30 minutes, because of its fast clearance from the circulation. NLP 10:0, CLP 9:1, and CLP 5:5-PEG exhibited long circulation times compared with free DOX. However, CLP 5:5, which exhibited the most positive zeta potential value, attained the lowest concentration after injection (even lower than the concentration of free DOX) 


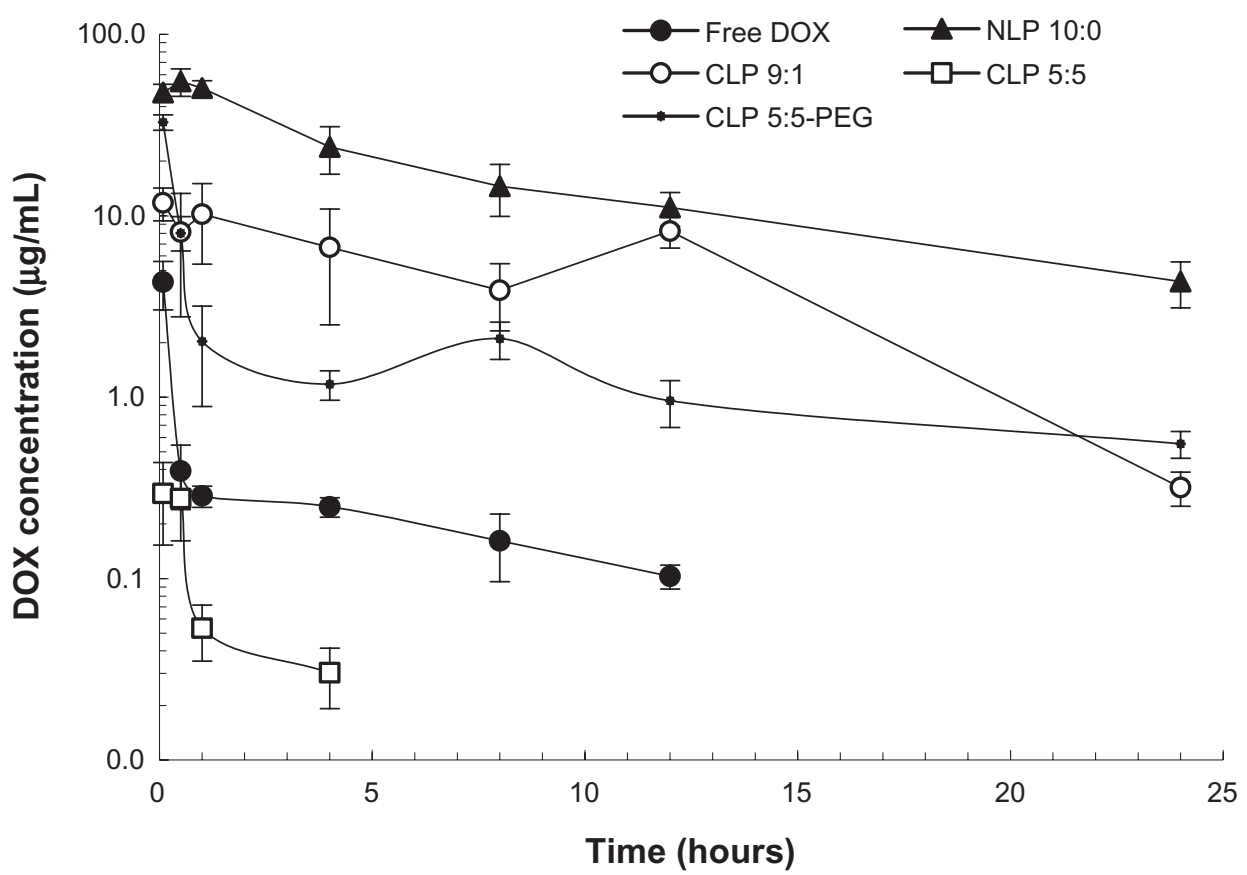

Figure 4 Plasma concentrations of doxorubicin (DOX) after intravenous injection of $5 \mathrm{mg} / \mathrm{kg}$ DOX-loaded liposomes or free DOX.

Note: The results represent the mean plus or minus the standard deviation from four to six mice per time point.

Abbreviations: CLP, cationic liposome; NLP, neutral liposome; PEG, polyethylene glycol.

and was not detected after 8 hours post injection. The phenomenon may be explained by the severe aggregation of CLP 5:5 with anionic species in the blood (see Figure 1), which accelerates the liposome clearance from the bloodstream. Among all the liposome preparations, NLP 10:0 attained the highest concentration after intravenous injection and the longest circulation time.

The pharmacokinetics parameters of the DOX-loaded liposomes and free DOX, which were calculated according to a noncompartmental model using WinNonlin software (WinNonlin Professional, v 3.1; Pharsight Corp., Mountain View, CA), are summarized in Table 2. Except for CLP 5:5, the DOX-loaded liposomes achieved longer performance in maximum plasma concentration $\left(\mathrm{C}_{\max }\right)$, area under the concentration-time curve (AUC), and mean residence time (MRT) than free DOX. Notably, NLP 10:0 exhibited the largest $\mathrm{AUC}_{0-\mathrm{t}}$ value. The samples ranked in $\mathrm{MRT}_{0-\mathrm{t}}$ as follows: NLP 10:0 > CLP 5:5-PEG > CLP 3:1 > free DOX $>$ CLP 5:5. However, regarding the elimination rate $(\lambda)$ from the bloodstream, CLP 5:5-PEG exhibited the slowest $\lambda\left(0.04\right.$ hours $\left.^{-1}\right)$ and longest half-life (18.06 hours), which implied a long circulation time.

The distributions of free DOX and four DOX-loaded liposomes in the blood, liver, spleen, lung, kidney, heart, and tumor that calculated from the AUC of different tissues on $0,0.083,0.5,1,4,8,12$, and 24 hours using the linear trapezoidal rule are presented in Figure 5. CLP 5:5 attained a dramatically high distribution in the lung and smaller distributions in the liver, spleen, kidney, blood, and tumor compared with the tissue distributions of NLP 10:0, CLP 9:1, and CLP 5:5-PEG. The phenomena were attributed to the lung capture of the aggregates of the anionic species in the

Table 2 Pharmacokinetic parameters of free doxorubicin (DOX) and DOX-loaded liposomes

\begin{tabular}{lllllll}
\hline Parameter* & Units & DOX & NLP 10:0 & CLP 9:I & CLP 5:5 & CLP 5:5-PEG \\
\hline $\mathrm{C}_{\max }$ & $\mu \mathrm{g} / \mathrm{mL}$ & 6.96 & 59.94 & 12.70 & 0.30 & 43.49 \\
$\lambda$ & $\mathrm{I} / \mathrm{h}$ & $0.1 \mathrm{I}$ & 0.08 & 0.15 & 0.19 & 0.04 \\
$\mathrm{t}_{1 / 2}$ & $\mathrm{~h}$ & 6.30 & 9.08 & 4.51 & 3.67 & 18.06 \\
$\mathrm{AUC}_{0-\mathrm{t}}$ & $\mathrm{h} \cdot \mu \mathrm{g} / \mathrm{mL}$ & 3.77 & 387.93 & 85.92 & 0.35 & 36.64 \\
$\mathrm{MRT}_{0-\mathrm{t}}$ & $\mathrm{h}$ & 3.13 & 6.68 & 5.59 & 0.98 & 6.19 \\
\hline
\end{tabular}

Note: *Parameters were calculated using WinNonlin software (WinNonlin Professional, v 3.I; Pharsight Corp., Mountain View, CA) and a noncompartmental model. Abbreviations: $\lambda$, first-order elimination rate constant; $A \cup C_{0-t}$, area under the concentration-time curve from the initial time to the time of the last observation, calculated using the linear trapezoidal rule; $C L P$, cationic liposome; $\mathrm{C}_{\max }$, maximum plasma concentration; $\mathrm{MRT}_{0-\mathrm{t}}$, mean residence time from the initial time to the time of the last measurable concentration; NLP, neutral liposome; PEG, polyethylene glycol; $\mathrm{t}_{1 / 2}$, half-life. 


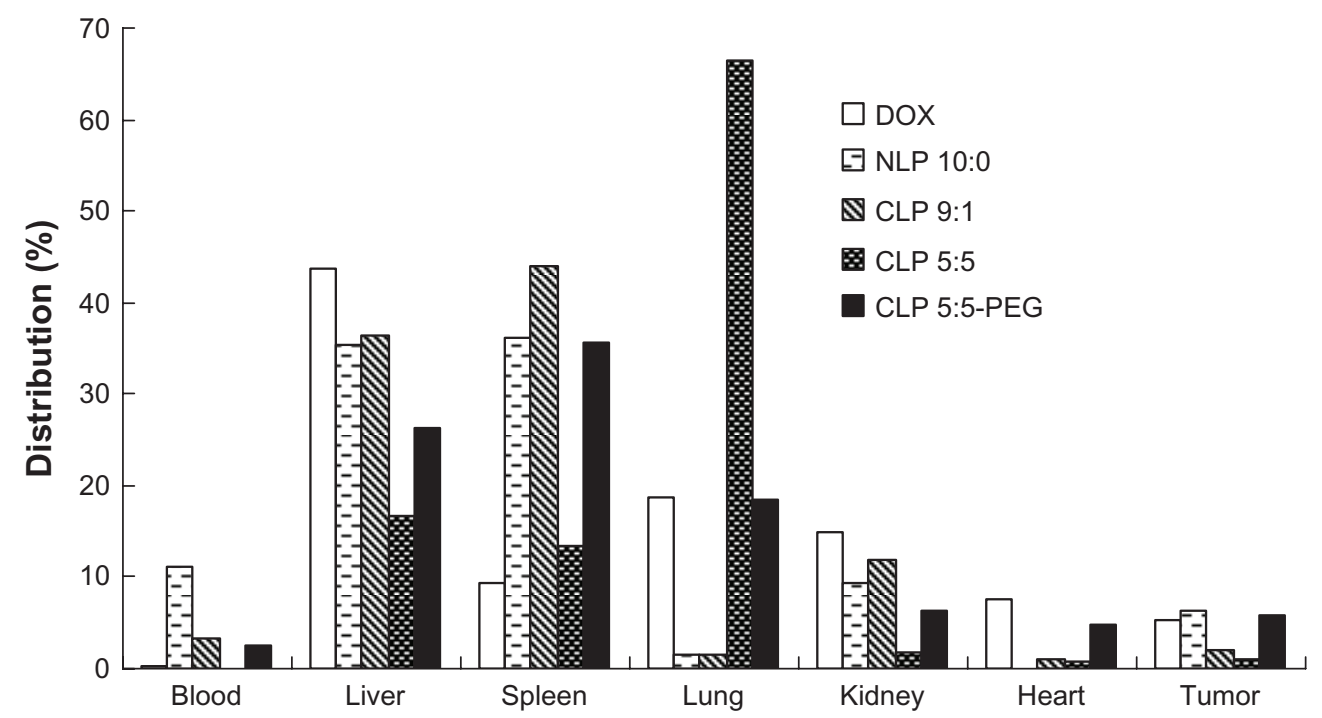

Figure 5 The distribution of doxorubicin (DOX)-loaded liposomes and free DOX in $\mathrm{H} 22$ tumor-bearing mice.

Notes: The distribution data were calculated using the linear trapezoidal rule from the area under the concentration-time curve of blood and different tissues at $0,0.083$, $0.5, I, 4,8,12$, and 24 hours. The results represent the mean value from six separate mice at each time points.

Abbreviations: CLP, cationic liposome; NLP, neutral liposome; PEG, polyethylene glycol.

blood and the CLPs. Regarding accumulation in the tumor, NLP 10:0 and CLP 5:5-PEG attained higher distributions than CLP 9:1 and CLP 5:5, which revealed that the NLPs and CLP-PEG were advantageous for enhancing the liposome accumulation in the tumor. When the positive surface potential is high, CLPs will readily aggregate in the serum (see Figure 1) and this will result in lung accumulation or embolism (see Figure 5), which is in accordance with some previous reports. ${ }^{12,15}$ The PEGylation of CLP 5:5 may shield the cationic sites on the liposomal surfaces and lower the surface potential; thus, CLP 5:5-PEG exhibited more stable profiles in vitro and in vivo and achieved better DOX accumulation in tumors than CLP 5:5.

\section{Therapeutic efficacy of liposomes}

Every DOX therapeutic group produced fewer tumors than the negative control group (treatment with saline) in the S180 tumor-bearing mice model $(P<0.01)$ (see Figure 6). The PEGylated liposomes (NLP-PEG and CLP-PEG) showed significant difference compared with the non-PEGylated liposomes (NLP and CLP) $(P<0.05)$ and saline $(P<0.01)$. The group treated with NLP 10:0-PEG exhibited the longest mean survival time. Comparing the comprehensive effect of every formulation, the authors conclude that the formulations rank in antitumor activities as follows: NLP 10:0-PEG $\geq$ CLP 3:1-PEG > CLP 3:1 $\approx$ NLP 10:0 $\approx$ free DOX $>$ saline. The PEGylation of NLPs and CLPs may enhance the antitumor effects for liposomes, with the best results obtained using NLP-PEG, although there was no significant difference between the NLP 10:0-PEG and CLP 3:1-PEG. It was anticipated that CLP 5:5, which exhibited the highest positive charge, would not exhibit good antitumor activity in this experiment because of the poor pharmacokinetic parameters and distribution in vivo (see Table 2 and Figure 5), so, for economical purposes, the authors only used CLP 3:1 and CLP 3:1-PEG.

The elimination of liposomes from the systemic circulation is a key issue affecting the therapeutic effect. PEGylation has been demonstrated to be an efficient approach to minimizing the interaction of conventional liposomes with the serum proteins and cells of the mononuclear phagocyte system in the liver and spleen, thus prolonging the plasma circulation time of the liposomes. ${ }^{32,33}$ Some researchers have reported that PEG concentrations of $\geq 6 \mathrm{~mol} \%$ shielded the electric surface potential of CLPs and that a higher concentration (15 mol\%) fully abolished the effect of charged groups on the liposome surface. ${ }^{34}$ NLP-PEG and CLP-PEG circulated longer and accumulated in the tumor in greater amounts because of the EPR effect. Subsequent to interacting with the endothelium through anionic sites on the luminal surfaces of the vessels, cationic drug carriers may be internalized by endothelial cells through endocytosis or extravasation via endothelial fenestrae. Despite the remarkable targeting of cationic liposomes to tumor vessels, this study found that a long circulation time in the bloodstream was still vital for achieving a good distribution in the tumor, because the NLPs and CLP-PEG achieved higher AUC and MRT values in the plasma (see Table 2) and delivered the 


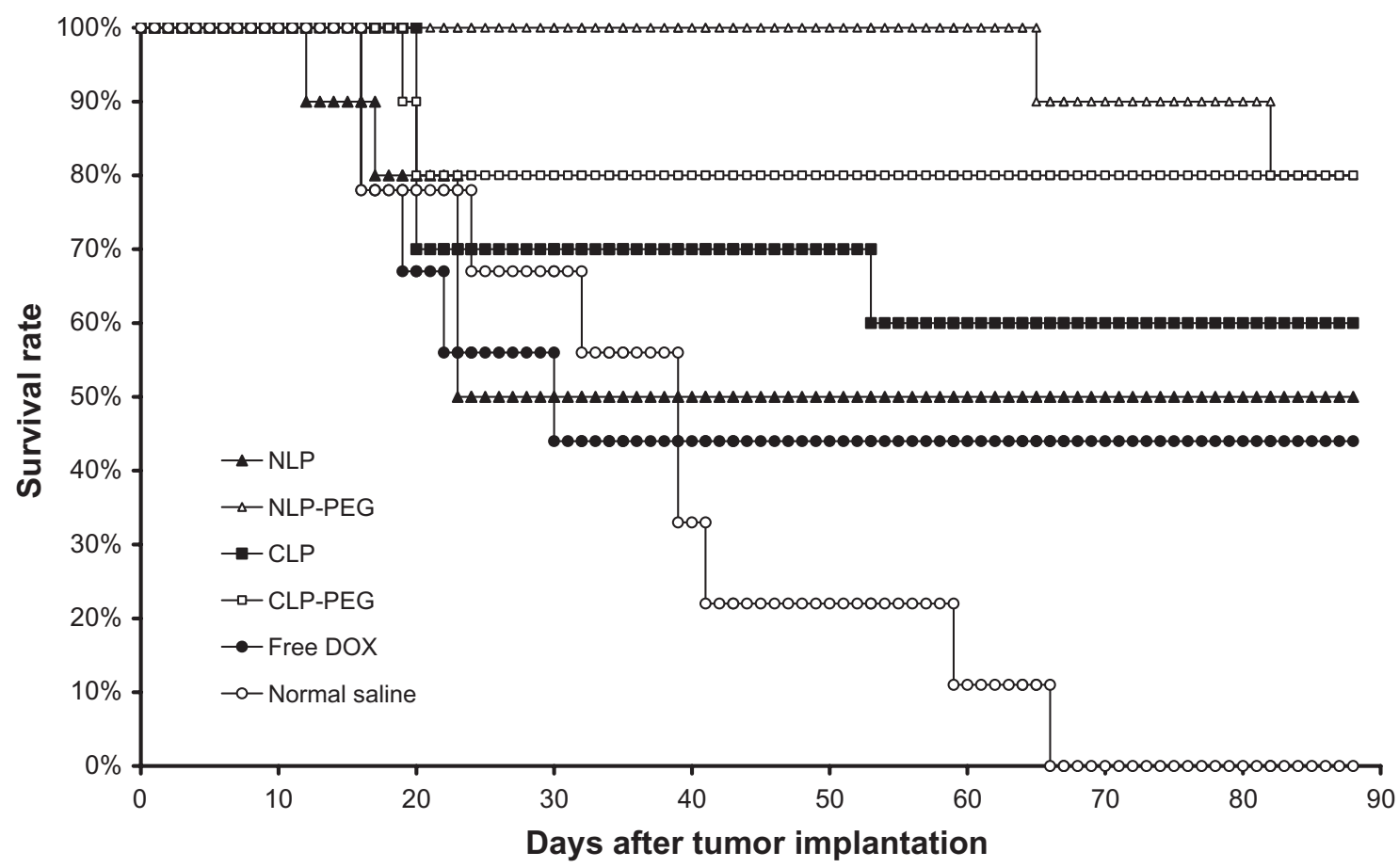

Figure 6 Mean survival time of female Kunming mice with sarcoma 180 xenografts after treatment. Dosage was adjusted to attain a doxorubicin (DOX) concentration of $5 \mathrm{mg} / \mathrm{kg}$ **

Note: *Data are presented as mean plus or minus standard deviation $(n=10)$.

Abbreviations: CLPs, cationic liposomes; CLP-PEG, polyethylene glycolylated cationic liposomes; NLPs, neutral liposomes; NLP-PEG, polyethylene glycolylated neutral liposomes.

largest amount of drug to the tumors (see Figure 5), whereas the CLP 5:5 exhibited high absorption of anionic proteins and were rapidly cleared from the circulation system (see Figures 1 and 5).

CLP-PEG have provided researchers with an opportunity to exploit the leaky tumor vasculature for cancer treatment. CLP-PEG may achieve better efficacy in suppressing tumor growth than conventional NLPs because disrupting small amounts of tumor vessels may induce embolism in tumor vessels, which deprives the tumor cells of nutrients and oxygen. The precondition was that the liposomes did not reduce the EPR effect. Apart from the prolonged circulation effects of PEG when anchored on the liposome surface, the ability to promote tumor vascular-specific uptake requires the ideal liposome to possess additional, specialized features. ${ }^{11}$ Therefore, caution should be exercised when using the strategy of targeting drug-loaded CLPs to the tumor vessels to improve drug and gene delivery in solid tumors.

\section{Conclusion}

Despite the remarkable targeting of tumor vessels through cationic molecules in vitro, this study found that a long circulation time in the bloodstream was still vital for achieving a good distribution in the tumor, because the NLPs and
CLP-PEG achieved high AUC and MRT values in vivo and delivered the largest amount of drug to the tumors, whereas the CLPs with high positive potential exhibited high adsorption of anionic proteins and were rapidly cleared from the circulation system. Studies revealed that the enhanced EPR effect exhibited by the PEGylated liposomes had a dominant effect on enhanced antitumor efficiency in vivo.

\section{Acknowledgments}

The National Natural Science Foundation of China (Nos 30772665 and 30970785) and the Doctoral Foundation of the Ministry of Education of China (No. 20100001110056) supported this work.

\section{Disclosure}

The authors report no conflicts of interest in this work.

\section{References}

1. Allen TM, Cullis PR. Drug delivery systems: entering the mainstream. Science. 2004;303(5665):1818-1822.

2. Obata Y, Tajima S, Takeoka S. Evaluation of pH-responsive liposomes containing amino acid-based zwitterionic lipids for improving intracellular drug delivery in vitro and in vivo. $J$ Control Release. 2010;142(2):267-276.

3. MacLachlan I, Cullis P, Graham RW. Progress towards a synthetic virus for systemic gene therapy. Curr Opin Mol Ther. 1999;1(2):252-259. 
4. Heyes J, Hall K, Tailor V, Lenz R, MacLachlan I. Synthesis and characterization of novel poly(ethylene glycol)-lipid conjugates suitable for use in drug delivery. J Control Release. 2006;112(2):280-290.

5. Ran S, Downes A, Thorpe PE. Increased exposure of anionic phospholipids on the surface of tumor blood vessels. Cancer Res. 2002;62(21):6132-6140.

6. Mounkes LC, Zhong W, Cipres-Palacin G, Heath TD, Debs RJ. Proteoglycans mediate cationic liposome-DNA complex-based gene delivery in vitro and in vivo. J Biol Chem. 1998;273(40):26164-26170.

7. Augustin HG, Kozian DH, Johnson RC. Differentiation of endothelial cells: analysis of the constitutive and activated endothelial cell phenotypes. Bioessays. 1994;16(12):901-906.

8. Strieth S, Eichhorn ME, Sauer B, et al. Neovascular targeting chemotherapy: encapsulation of paclitaxel in cationic liposomes impairs functional tumor microvasculature. Int J Cancer. 2004;110(1):117-124.

9. Strieth S, Eichhorn ME, Alexander W, et al. Targeted liposomal paclitaxel raises tumor vessel leakiness. Otolaryngol Head Neck Surg. 2008;139(2 Suppl 1):P94.

10. Wu J, Lee A, Lu Y, Lee RJ. Vascular targeting of doxorubicin using cationic liposomes. Int J Pharm. 2007;337(1-2):329-335.

11. Abu Lila AS, Kizuki S, Doi Y, Suzuki T, Ishida T, Kiwada H. Oxaliplatin encapsulated in PEG-coated cationic liposomes induces significant tumor growth suppression via a dual-targeting approach in a murine solid tumor model. J Control Release. 2009;137(1):8-14.

12. Campbell RB, Fukumura D, Brown EB, et al. Cationic charge determines the distribution of liposomes between the vascular and extravascular compartments of tumors. Cancer Res. 2002;62(23):6831-6836.

13. Cullis PR, Chonn A, Semple SC. Interactions of liposomes and lipidbased carriers with blood proteins: relation to clearance behavior in vivo. Adv Drug Deliv Rev. 1998;32(1-2):3-17.

14. Stamatatos L, Leventis R, Zuckermann MJ, Silvius JR. Interactions of cationic lipid vesicles with negatively charged phospholipid vesicles and biological membranes. Biochemistry. 1988;27(11):3917-3925.

15. Litzinger DC, Brown JM, Wala I, et al. Fate of cationic liposomes and their complex with oligonucleotide in vivo. Biochim Biophys Acta. 1996;1281(2):139-149.

16. McLean JW, Fox EA, Baluk P, et al. Organ-specific endothelial cell uptake of cationic liposome-DNA complexes in mice. Am J Physiol. 1997;273(1 Pt 2):H387-H404.

17. Dass CR. Improving anti-angiogenic therapy via selective delivery of cationic liposomes to tumour vasculature. Int J Pharm. 2003; 267(1-2):1-12.

18. Zhang Y, Qi XR, Gao Y, Wei SL, Maitani Y, Nagai T. Mechanisms of co-modified liver-targeting liposomes as gene delivery carriers based on cellular uptake and antigens inhibition effect. $J$ Control Release. 2007;117(2):281-290.

19. Roy S, Mohanty A, Dey J. Microviscosity of bilayer membranes of some $\mathrm{N}$-acylamino acid surfactants determined by fluorescence probe method. Chem Phys Lett. 2005;414(1-3):23-27.

20. Centra M, Ratych RE, Cao GL, et al. Culture of bovine pulmonary artery endothelial cells on Gelfoam blocks. FASEB J. 1992; 6(12):3117-3121.
21. Gao X, Huang L. A novel cationic liposome reagent for efficient transfection of mammalian cells. Biochem Biophys Res Commun. 1991;179(1):280-285.

22. Shinozawa S, Mimaki Y, Araki Y, Oda T. Determination of the concentration of adriamycin and its metabolites in the serum and tissues of Ehrlich carcinoma-bearing mice by high-performance liquid chromatography. J Chromatogr. 1980;196(3):463-469.

23. Qi XR, Maitani Y, Nagai T, Wei SL. Comparative pharmacokinetics and antitumor efficacy of doxorubicin encapsulated in soybean-derived sterols and poly(ethylene glycol) liposomes in mice. Int J Pharm. 1997;146(1):31-39.

24. Uchiyama K, Nagayasu A, Yamagiwa Y, Nishida T, Harashima H, Kiwada H. Effects of the size and fluidity of liposomes on their accumulation in tumors: a presumption of their interaction with tumors. Int J Pharm. 1995;121(2):195-203.

25. Qi XR, Maitani Y, Nagai T. Relation of rigidity of membrane and stability of dipalmitoylphosphatidylcholine liposomes with soybeanderived sterols prepared by reverse-phase evaporation vesicle method. Chem Pharm Bull. 1996;44(1):237-240.

26. Zhao W, Ma HL, Qi XR. Cationic liposomes loaded with doxorubicin targeting to the tumor neovasculature in vitro. Yao Xиe Xие Bao. 2007;42(9):982-988. Chinese.

27. Silvander M, Johnsson M, Edwards K. Effects of PEG-lipids on permeability of phosphatidylcholine/cholesterol liposomes in buffer and in human serum. Chem Phys Lipids. 1998;97(1):15-26.

28. Johnstone SA, Masin D, Mayer L, Bally MB. Surface-associated serum proteins inhibit the uptake of phosphatidylserine and poly(ethylene glycol) liposomes by mouse macrophages. Biochim Biophys Acta. 2001;1513(1):25-37.

29. Noguchi A, Furuno T, Kawaura C, Nakanishi M. Membrane fusion plays an important role in gene transfection mediated by cationic liposomes. FEBS Lett. 1998;433(1-2):169-173.

30. Elouahabi A, Ruysschaert JM. Formation and intracellular trafficking of lipoplexes and polyplexes. Mol Ther. 2005;11(3):336-347.

31. Torchilin VP. Fluorescence microscopy to follow the targeting of liposomes and micelles to cells and their intracellular fate. Adv Drug Deliv Rev. 2005;57(1):95-109.

32. Lu WL, Qi XR, Zhang Q, et al. A pegylated liposomal platform: pharmacokinetics, pharmacodynamics, and toxicity in mice using doxorubicin as a model drug. J Pharmacol Sci. 2004;95(3):381-389.

33. Abu Lila AS, Ishida T, Kiwada H. Recent advances in tumor vasculature targeting using liposomal drug delivery systems. Expert Opin Drug Deliv. 2009;6(12):1297-1309.

34. Levchenko TS, Rammohan R, Lukyanov AN, Whiteman KR, Torchilin VP. Liposome clearance in mice: the effect of a separate and combined presence of surface charge and polymer coating. Int J Pharm. 2002;240(1-2):95-102.
International Journal of Nanomedicine

\section{Publish your work in this journal}

The International Journal of Nanomedicine is an international, peerreviewed journal focusing on the application of nanotechnology in diagnostics, therapeutics, and drug delivery systems throughout the biomedical field. This journal is indexed on PubMed Central, MedLine, CAS, SciSearch ${ }^{\circledR}$, Current Contents ${ }^{\circledR} /$ Clinical Medicine,

\section{Dovepress}

Journal Citation Reports/Science Edition, EMBase, Scopus and the Elsevier Bibliographic databases. The manuscript management system is completely online and includes a very quick and fair peer-review system, which is all easy to use. Visit http://www.dovepress.com/ testimonials.php to read real quotes from published authors. 